\title{
SCHUR INDICES IN FINITE QUATERNION-FREE GROUPS
}

\author{
A. D. $\mathrm{OH}$
}

\begin{abstract}
Let $G$ be a finite, quaternion-free group with exponent $e$, let $F$ be a field of characteristic zero and let $\chi$ be an absolutely irreducible character of $G$. Suppose that a Sylow 2-subgroup of the Galois group of $F(\sqrt[e]{1})$ over $F$ is cyclic. It is shown that if $\chi$ is not real valued, then the Schur index of $\chi$ over $F$ is odd.
\end{abstract}

Let $G$ be a finite group with exponent $e$ and let $F$ be a field of characteristic zero. We denote the set of absolutely irreducible characters of $G$ by $\operatorname{Irr}(G)$, and write $m_{F}(\chi)$ for the Schur index over $F$ of $\chi \subset \operatorname{Irr}(G) . \xi_{n}$ denotes a primitive $n$th root of unity. We say that $\chi \in \operatorname{Irr}(G)$ is real valued if the field $Q(\chi)$ is embedded in $R$, the field of real numbers. Following [6] we call $G$ a quaternion-free group if a generalized quaternion group is not involved in $G$.

In [3], B. Fein proved that if -1 is a sum of two squares in $F$ and a Sylow 2-subgroup of the Galois group $\operatorname{Gal}\left(F\left(\xi_{e}\right) / F\right)$ is cyclic, then $m_{F}(\chi)$ is odd. Because of the well-known Brauer-Speiser theorem, we investigate $m_{F}(\chi)$ when $\chi$ is not real valued. We prove the following.

THEOREM. Let $G$ be a finite, quaternion-free group with exponent $e$, let $F$ be a field of characteristic zero and let $\chi \in \operatorname{Irr}(G)$. Suppose that a Sylow 2-subgroup of the Galois group $\operatorname{Gal}\left(F\left(\xi_{e}\right) / F\right)$ is cyclic. If $\chi$ is not real valued, then $m_{F}(\chi)$ is odd.

The assumption that $G$ is quaternion-free in the theorem cannot be dropped as the following example shows. Let $G$ be a nilpotent group of order $8 q$ where $q$ is a prime such that $q \equiv 7(\bmod 8)$, and assume that its Sylow 2-subgroup is generalized quaternion. Choose a faithful, complex irreducible character $\chi$ of $G$ and let $F=Q(\chi)$. Clearly, $F=Q\left(\xi_{q}\right)$ and $F\left(\xi_{e}\right)=F(\sqrt{-1})$. To see that $m_{F}(\chi)=2$, we consider an $F$-triple $(G, X, \chi)$ where $X \leqslant G$ with $|G: X|=2$. Since $q \equiv 7(\bmod 8),-1$ is not a norm in $F(\sqrt{-1}) / F$ by [1, Note, p. 173], thus $m_{F}(\chi)=2$ by Theorem 2.2 of [2].

The proof of the theorem is based on reductions of $G$ and $\chi$ to an $F$-triple as in [2], and norm computations in reduced field extensions utilizing Theorem 2.2 of [2].

LEMMA 1. If $(H, X, \theta)$ is an F-triple and $K$ is a subfield of $F(\theta)$, then $(H, X, \theta)$ is a K-triple.

Proof. If $K$ is a subfield of $F$, then $(H, X, \theta)$ is a $K$-triple by Lemma 9.17 (b) of [1]. Since $(H, X, \theta)$ is an $F(\theta)$-triple, the result follows.

Received by the editors December 27, 1981.

1980 Mathematics Subject Classification. Primary $20 \mathrm{C} 15$. 
Lemma 2. Let $(H, X, \theta)$ be an F-triple. Suppose that $H / X$ is a cyclic 2-group and $|X \cap Y|=2$ for a cyclic supplement $Y$ to $X$ in $H$. Then there is an F-triple $\left(Y X_{0}, X_{0}, \theta_{0}\right)$ with $X_{0} \leqslant X$ and $F\left(\theta_{0}\right) \subseteq F(\theta)$, such that (i) $X_{0}$ is a 2-group or $\left|X_{0}\right|=2 q$ for an odd prime $q$, (ii) $Y \cap X=Y \cap X_{0}$, and (iii) $m_{F}(\theta)=1$ if $m_{F}\left(\theta_{0}\right)=$ 1.

Proof. Let $\lambda$ be an irreducible constituent of $\theta_{X}$, and write $\pi(X)$ for the set of primes dividing $|X|$. By Lemma 2.1 of [2], $\operatorname{Gal}(F(\lambda) / F(\theta))$ is a cyclic 2-group, thus $\left\{F(\theta)\left(\xi_{q^{i}}\right): q \in \pi(X)\right.$ and $\left.q^{i} \||X|\right\}$ is linearly ordered; moreover, $F(\theta)\left(\xi_{q^{i}}\right)=$ $F(\theta)\left(\xi_{q}\right)$ if $q \in \pi(X)$ is odd and $i>1$. Thus we have either (a) or (b) below;

(a) $F(\lambda)=F(\theta)\left(\xi_{q}\right)$ for some odd prime $q \in \pi(X)$.

(b) $F(\lambda)=F(\theta)\left(\xi_{s}\right)$ where $s$ is the order of a Sylow 2-subgroup of $X$.

In case (a), define $X_{0}=A(X \cap Y)$ where $A \leqslant X$ with $|A|=q$, so that $\left|X_{0}\right|=2 q$ and $Y \cap X=Y \cap X_{0}$; in case (b), define $X_{0}$ to be a Sylow 2-subgroup of $X$. Letting $\theta_{0}=\theta_{Y X_{0}}$, we see that $\left(Y X_{0}, X_{0}, \theta_{0}\right)$ is an $F(\theta)$-triple; hence it is an $F$-triple by Lemma 1. Since $F(\lambda)=F(\theta)\left(\lambda_{X_{0}}\right)$, Theorem 2.2 of [2] implies that $m_{F}(\theta)=1$ if and only if $m_{F(\theta)}\left(\theta_{0}\right)=1$. Thus, $m_{F}(\theta)=1$ if $m_{F}\left(\theta_{0}\right)=1$.

Proof of Theorem. By Goldschmidt-Isaacs Theorem [2, Theorem 1] and $m_{F}(\chi)$ $=m_{F(\chi)}(\chi)$, we may assume $\sqrt{-1} \notin F$ and $F=F(\chi)$. Suppose that $2 \mid m_{F}(\chi)$. Then, by Theorem 2 of [2], there exists an $F$-triple $(H, X, \theta)$ such that $H$ is a section of $G$, $H / X$ is a 2-group, $2 \mid m_{F}(\theta)$ and $2 \nmid|F(\theta): F|$. Note that $H / X$ is cyclic since $H / X$ is a section of $\operatorname{Gal}\left(F\left(\xi_{e}\right) / F\right)$ by Lemma 2.1 of [2], and since $\operatorname{Gal}\left(F\left(\xi_{e}\right) / F\right)$ has a cyclic Sylow 2-subgroup.

Let $Y$ be a cyclic supplement to $X$ in $H$ such that $X \cap Y$ is a 2-group. Since $\theta \in \operatorname{Irr}(H)$ is faithful and $X \cap Y$ is central in $H, F(\theta)$ contains a primitive $|X \cap Y|$ th root of unity and thus $|X \cap Y| \leqslant 2$. The case $|X \cap Y|=1$ does not occur by Theorem 2.2 of [2]. Hence $|X \cap Y|=2$. Now, by Lemma 2 we reduce $(H, X, \theta)$ to the case where $X$ is a 2-group or $|X|=2 q$ for an odd prime $q$.

If $X$ is a 2-group, then Theorem 2.13 of [6] implies that a generalized quaternion group is involved in $H$ (hence in $G$ ), against our assumption on $G$.

Next, suppose $|X|=2 q$ and assume $|Y|=4$. Put $F_{1}=F \cap Q\left(\xi_{e}\right)$ and let $\lambda$ be an irreducible constituent of $\theta_{X}$. Then $(H, X, \theta)$ is an $F_{1}$-triple (by Lemma 1) and $2 \mid m_{F_{1}}(\theta)$; it follows from Theorem 2.2 of [2] that -1 cannot be a norm in $F_{1}(\lambda) / F_{1}(\theta)$. On the other hand, we observe that $\left|F_{1}(\lambda): F_{1}(\theta)\right|=2$ (by Lemma 2.1 of [2]), $\sqrt{-1} \notin F_{1}(\theta)$ (since $|F(\theta): F|$ is odd) and that $F_{1}(\theta)(\sqrt{-1})=F_{1}(\theta)\left(\xi_{q}\right)=$ $F_{1}(\lambda)$ (since $\operatorname{Gal}\left(Q\left(\xi_{e}\right) / F_{1}\right)$ has a cyclic Sylow 2-subgroup). Put $K=F_{1}(\theta)$ and $L=F_{1}(\lambda)$. We compute the norm residue symbol $(-1, L / K)_{\mathfrak{p}}$ where $\mathfrak{p}$ is a $K$-prime. Since $\chi$ is not real valued, $F_{1} \nsubseteq R$ and thus $(-1, L / K)_{\mathfrak{p}}=1$ if $\mathfrak{p}$ is infinite; since -1 is a unit, $(-1, L / K)_{\mathfrak{p}}=1$ if $\mathfrak{p}$ is a finite $K$-prime unramified in $L / K$. If $\mathfrak{p}$ is a finite $K$-prime ramified in $L / K$ then Corollary 6-2-4 of [4] implies that $\mathfrak{p}$ divides $q$ since $L=K\left(\xi_{q}\right)$, and that $\mathfrak{p}$ divides 2 since $L=K(\sqrt{-1})$; this is impossible. Hence $(-1, L / K)_{\mathfrak{b}}=1$ for all $K$-primes $\mathfrak{p}$, and the norm theorem [5, p. 392] implies that -1 is a norm in $L / K$, a contradiction.

For the remaining case where $|X|=2 q$ and $|Y|>4$, the following Lemma yields a contradiction, which will complete the proof of Theorem. 
LEMMA. Let $(H, X, \theta)$ be an $F$-triple such that $H=Y X$ for a cyclic 2-group $Y$ with $|X \cap Y|=2$ and that $|X|=2 q$ for an odd prime $q$. Suppose that $\operatorname{Gal}\left(F\left(\xi_{n}\right) / F\right)$ has a cyclic Sylow 2-subgroup, where $n$ is the exponent of $H$. If $|Y|>4$, then $m_{F}(\theta)=1$.

Proof. Let $\lambda$ be an irreducible constituent of $\theta_{X}$. We will show that -1 is a norm in $F_{0}(\lambda) / F_{0}(\theta)$ for a subfield $F_{0}$ of $F$; it follows then by Theorem 2.2 of [2] and Lemma 1 that $m_{F_{0}}(\theta)=1$, and hence $m_{F}(\theta)=1$.

Let $|H|=2^{t} q$. By Lemma 2.1 of $[2],|F(\lambda): F(\theta)|=2^{t-1}$. Since $\operatorname{Gal}\left(F\left(\xi_{n}\right) / F\right)$ has a cyclic Sylow 2-subgroup and $\left|F(\theta)\left(\xi_{2^{t}}\right): F(\theta)\right| \leqslant 2^{t-1}$, it follows that $\xi_{2^{t}} \in$ $F(\lambda)$ and hence $F(\lambda)=F\left(\xi_{q}\right)=F\left(\xi_{n}\right)$. If $q \equiv 3(\bmod 4)$, Lemma 2.1 of [2] would yield $t<3$, which is not the case. Thus $q \equiv 1(\bmod 4)$. Set $F_{0}=F \cap Q\left(\xi_{n}\right)$, and write $K=F_{0}(\lambda), k=F_{0}(\theta)$; so the extension $K / k$ is cyclic of degree $2^{t-1}$ by Lemma 2.1 of [2] and Lemma 1 .

Now, we show that -1 is a norm in $K / k$, i.e., that the norm residue symbol $(-1, K / k)_{\mathfrak{p}}=1$ for all $k$-primes $\mathfrak{p}$. It follows from $F(\lambda)=F\left(\xi_{q}\right)=F\left(\xi_{n}\right)$ that

$$
\operatorname{Gal}\left(Q\left(\xi_{n}\right) / F_{0}\right) \cong \operatorname{Gal}(F(\lambda) / F) \cong \operatorname{Gal}\left(Q\left(\xi_{q}\right) / F \cap Q\left(\xi_{q}\right)\right)
$$

and $K=k\left(\xi_{q}\right)=k\left(\xi_{n}\right)$. Note that 4 divides $|F(\lambda): F|$, and $\operatorname{Gal}\left(Q\left(\xi_{n}\right) / F_{0}\right)$ is cyclic; but, the structure of $\operatorname{Gal}\left(Q\left(\xi_{n}\right) / Q\right)$ shows that if $E$ is a subfield of $Q\left(\xi_{n}\right) \cap R$ with $\left|Q\left(\xi_{n}\right): E\right|=4$, then $\operatorname{Gal}\left(Q\left(\xi_{n}\right) / E\right)$ is a four-group. Hence $F_{0} \nsubseteq R$; and thus $(-1, K / k)_{\mathfrak{p}}=1$ for infinite primes $\mathfrak{p}$ of $k$. Thus by Corollary 6-2-4 of [4], we need only show that $(-1, K / k)_{\mathfrak{p}}=1$ for finite $k$-primes $\mathfrak{p}$ dividing $q$. Since $q \equiv 1$ $(\bmod 4), \sqrt{-1} \in Q_{q}$. By Goldschmidt-Isaacs Theorem [2, Theorem 1], we have $m_{k_{\mathfrak{p}}}(\theta)=1$ and thus $(-1, K / k)_{\mathfrak{p}}=1$. This completes the proof of Lemma.

\section{REFERENCES}

1. I. M. Isaacs, Character theory of finite groups, Academic Press, New York, 1976.

2. D. M. Goldschmidt and I. M. Isaacs, Schur indices in finite groups, J. Algebra 33 (1975), 191-199.

3. B. Fein, Schur indices and sums of squares, Proc. Amer. Math. Soc. 51 (1975), 31-34.

4. L. J. Goldstein, Analytic number theory, Prentice-Hall, Englewood Cliffs, N. J., 1971.

5. S. Iyanaga, The theory of numbers, North-Holland, Amsterdam, 1975.

6. L. Dornhoff, M-groups and 2-groups, Math. Z. 100 (1967), 226-256.

Department of Mathematics, Stillman College, Tuscaloosa, Alabama 35401 